\title{
Profile and clinical implication of circular RNAs in human papillary thyroid carcinoma
} \author{
, Gang Yuan Corresp. 1 \\ ${ }^{1}$ Department of Internal Medicine, Tongji Hospital, Huazhong University of Science and Technology, Wuhan, China \\ 2 Molecular Diagnostic Laboratory, Tongji Hospital, Huazhong University of Science and Technology, Wuhan, China \\ Corresponding Authors: Daowen Wang, Gang Yuan \\ Email address: dwwang@tjh.tjmu.edu.cn, gangyuan@tjh.tjmu.edu.cn
}

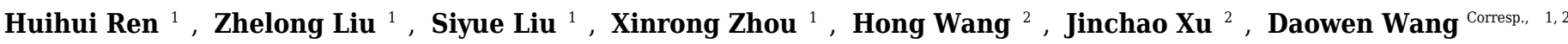

Background: Differently expressed circular RNAs (circRNAs) have been reported to play a considerable role in tumor behavior; however, the expression profile and biological function of circRNAs in papillary thyroid carcinoma (PTC) remains unknown. Thus, the study was aimed to characterize the circRNA expression profile to comprehensively understand the biological behavior of PTC. Methods : We investigated the expression profile of circRNAs using circRNA microarray in three pairs of PTC and adjacent normal tissues. Quantitative real-time reverse transcription polymerase chain reaction (qRT-PCR) was used to validate eight candidate circRNAs in 40 paired PTC tumors and adjacent normal samples. Next, we employed a bioinformatics tool to identify putative miRNA and circRNA-associated downstream genes, followed by constructing a network map of circRNA-miRNA-mRNA interactions and exploring the potential role of the candidate circRNAs. Results: In total, 206 up- and 177 downregulated circRNAs were identified in PTC tissues (fold change $>1.5 ; P<0.05$ ). The expression levels of eight candidate circRNAs confirmed by qRT-PCR were significantly different between the PTC and normal samples. The downstream genes of candidate circRNAs participated in various biological processes and signaling pathways. The most up and downregulated circRNAs were hsa_circRNA_007148 and hsa_circRNA_047771. The lower expression level of hsa_circRNA_047771 was associated BRAFV600 mutation, lymph node metastasis (LNM), as well as with advanced TNM stage (all $\mathrm{P}<0.05$ ). The higher expression level of hsa_circRNA_007148 was significantly correlated with LNM $(P<0.05)$. The areas under receiver operating curve were $0.876(95 \% \mathrm{Cl}, 0.78-0.94)$ for hsa_circRNA_047771 and $0.846(95 \% \mathrm{Cl}, 0.75-0.96)$ for hsa_circRNA_007148. Discussion: The study suggests that dysregulated circRNAs play a critical role in PTC pathogenesis. PTC-related hsa_circRNA_047771 and hsa_circRNA_007148 may serve as potential diagnostic biomarkers and prognostic predictors for PTC patients. 
1 Profile and Clinical Implication of Circular RNAs in Human Papillary Thyroid Carcinoma

2 Huihui Ren ${ }^{1 \#}$, Zhelong Liu ${ }^{1 \#,}$, Siyue Liu ${ }^{1}$ Xinrong Zhou ${ }^{1}$, Hong Wang ${ }^{2}$, Jinchao Xu${ }^{2}$, Daowen

3

4

8 \#co-authors

$9 \quad{ }^{*}$ Corresponding author

10 Gang Yuan, MD, $\mathrm{PhD}$, and Daowen Wang, $\mathrm{MD}, \mathrm{PhD}$

11 Department of Internal Medicine, Tongji Hospital, Tongji Medical College, Huazhong

12 University of Science \& Technology, Wuhan 430030, China

13 Tel and Fax: +86-27-8366-2883

14 Email: gangyuan@tjh.tjmu.edu.cn and dwwang@tjh.tjmu.edu.cn 


\section{Abstract}

Background: Differently expressed circular RNAs (circRNAs) have been reported to play a considerable role in tumor behavior; however, the expression profile and biological function of circRNAs in papillary thyroid carcinoma (PTC) remains unknown. Thus, the study was aimed to characterize the circRNA expression profile to comprehensively understand the biological behavior of PTC.

Methods: We investigated the expression profile of circRNAs using circRNA microarray in three pairs of PTC and adjacent normal tissues. Quantitative real-time reverse transcriptionpolymerase chain reaction (qRT-PCR) was used to validate eight candidate circRNAs in 40 paired PTC tumors and adjacent normal samples. Next, we employed a bioinformatics tool to identify putative miRNA and circRNA-associated downstream genes, followed by constructing a network map of circRNA-miRNA-mRNA interactions and exploring the potential role of the candidate circRNAs.

Results: In total, 206 up- and 177 downregulated circRNAs were identified in PTC tissues (fold change $>1.5 ; \mathrm{P}<0.05)$. The expression levels of eight candidate circRNAs confirmed by qRTPCR were significantly different between the PTC and normal samples. The downstream genes of candidate circRNAs participated in various biological processes and signaling pathways. The most up and downregulated circRNAs were hsa_circRNA_007148 and hsa_circRNA_047771.

The lower expression level of hsa_circRNA_047771 was associated BRAFV600 mutation, lymph node metastasis (LNM), as well as with advanced TNM stage (all $\mathrm{P}<0.05$ ). The higher 
35 expression level of hsa_circRNA_007148 was significantly correlated with LNM $(\mathrm{P}<0.05)$. The

36 areas under receiver operating curve were 0.876 (95\% CI, 0.78-0.94) for hsa_circRNA_047771

37 and $0.846(95 \% \mathrm{CI}, 0.75-0.96)$ for hsa_circRNA_007148.

38 Discussion: The study suggests that dysregulated circRNAs play a critical role in PTC

39 pathogenesis. PTC-related hsa_circRNA_047771 and hsa_circRNA_007148 may serve as

40 potential diagnostic biomarkers and prognostic predictors for PTC patients. 


\section{Introduction}

42 Thyroid cancer is the most common malignant tumor of the endocrine organs, whose morbidity

43 has continuously increased in recent decades(La Vecchia et al. 2015; Siegel et al. 2017). The

44 dominant histological type is papillary thyroid carcinoma (PTC), which accounts for $85-95 \%$ of

45 all cases(La Vecchia et al. 2015). Although many studies have been performed to better

46 understand PTC, controversy remains regarding the malignancy diagnosis, postoperative

47 treatment, and prognosis in patients with thyroid nodules. Thyroid fine-needle aspiration biopsy

48 (FNAB) is a standard method for the preoperative evaluation of thyroid nodules, up to $30 \%$ of

49 nodules with indeterminate cytology; however, it cannot be definitively diagnosed(Haugen

50 2017). Operative treatment was suggested for most of these patients, although only $10-20 \%$ of

51 nodules were diagnosed as malignant lesions. An urgent clinical challenge is to develop an

52 effective diagnostic method to reduce overdiagnosis and overtreatment.

53 Recent studies have provided evidence that genetic factors may play a key role in the

54 development of thyroid cancer. The BRAFV600E mutation, resulting in activation of MAPK

55 pathway signaling, is significantly associated with more aggressive characteristics of PTCs and

56 is of great importance in risk-stratification and the management of patients with thyroid

57 nodules(Kwak et al. 2009; Xing 2007). In addition, various noncoding RNAs (ncRNAs), such as microRNAs (miRNAs) and linear noncoding RNAs (lncRNAs), also participate in the progression and pathogenesis of PTC(Fuziwara \& Kimura 2016; Liyanarachchi et al. 2016).

60 However, the genomic and epigenomic mechanisms in thyroid cancer pathogenesis have not 
61 been clarified.

62 Circular RNAs (circRNAs), a novel class of endogenous noncoding RNAs, have become a

63 research hotspot in the cancer field. Circular RNAs, unlike linear RNA, have no poly(A) tails

64 and 5' caps, are characterized by a covalently closed loop structure formed by a tail $3^{\prime}$ splice site

65 and a head 5' splice site(Jeck \& Sharpless 2014). Recently, circRNAs were found to be highly

66 conserved in sequence, maintained stability in mammalian cells, and showed tissue-specific

67 expression(Chen \& Schuman 2016; Rybak-Wolf et al. 2015). These properties make circRNAs

68 potential molecular biomarkers for multiple diseases. Furthermore, circRNAs are important

69 transcriptional regulators of gene expression involved in the pathogenesis of various diseases.

70 For example, circRNAs could function as miRNA sponges to compete with endogenous RNA,

71 regulating the expression of target genes(Zhao \& Shen 2015). A human circRNA, ciRS-7

72 transcript (antisense to the cerebellar degeneration-related protein), acts as an miRNA-7 sponge

73 by binding to the microRNA effector complexes, which inactivate the function of miRNA(Lukiw

74 2013). Mounting evidence has demonstrated that circRNAs play a significant role in

75 carcinogenesis, acting as oncogenes or onco-suppressors. Differentially expressed circRNAs

76 were observed in various types of cancers and were associated with the development, invasion

77 and metastasis of human tumors(Chen et al. 2017; Han et al. 2017; Meng et al. 2017; Wu et al.

78 2010). These lines of evidence have suggested that circRNAs can be potential therapeutic targets

79 for diseases.

80 Although the association between circRNAs and various human cancers has been extensively 
81 evaluated and well established, the diagnostic value and biological function of circRNAs in PTC

82 remain largely elusive. Until now, the only study focused on this issue was reported by Peng et

83 al.(Peng et al. 2017) and included 18 thyroid samples (six PTC, six paired contralateral normal

84 samples, and six benign thyroid lesions). Although a role of circRNAs in PTC pathogenesis was

85 observed, the clinical value of circRNAs for PTC diagnosis, therapy, and prognosis remains

86 undefined. The present study explored the circRNA expression profile in PTC and paired normal

87 tissues and examined the fundamental role of circRNAs in PTC, contributing not only to the

88 understanding of tumorigenesis but also to improving the diagnosis and management of thyroid

89 cancer. 
Materials and methods

91

92

\section{Patients and tissue samples}

This study was approved by the Ethics Committee of Tongji Hospital, Tongji Medical, College, Huazhong University of Science and Technology (RB approval number, TJ-C20150806).

Written consent was obtained from the patients before surgery. Forty PTC and matched adjacent noncancerous tissue samples were obtained from patients who had undergone thyroidectomy at the Department of General Surgery at Tongji Hospital Affiliated Huazhong University of Science and Technology (Wuhan, Hubei province, China) from September 2016 to February 2017. All tissue samples were immediately soaked in RNAlater Reagent (Servicebio, Wuhan, China) and were preserved at $-80^{\circ} \mathrm{C}$ until further use. Of these samples, three pairs were used for circRNA microarray analysis, and 40 samples (including the remaining 37 samples and the 3 samples used for microarray analysis) were used for additional validation in the present study. The general clinicopathologic characteristics of the participants are shown in Table 1. None of the participants included in this study underwent radiotherapy and chemotherapy preoperatively.

\section{Total RNA isolation and quality control}

Total RNA was isolated from PTC and paired adjacent normal tissues using a DNA/RNA coextraction kit (Tiangen Biotech, Beijing, China; DP121221) according to the manufacturer's protocol. The purity and concentration of the total RNA samples were qualified and quantified using the NanoDrop ND1000 system (NanoDrop Technologies/Thermo Scientific, Wilmington, DE, USA). For spectrophotometry, the O.D. A260/A280 ratio should be close to 2.0 for pure 
110 RNA. The O.D. A260/A230 ratio should be more than 1.8. The RNA integrity was assessed by

111 electrophoresis using a denaturing agarose gel.

\section{Microarray analysis}

113 The microarray analysis of circRNAs was performed based on the Arraystar's standard protocols

114 (Arraystar, Rockville, MD). Briefly, total RNA was digested with RNase R (Epicentre, Inc.) to

115 remove linear RNAs and enrich circular RNAs. Next, the enriched circular RNAs were amplified

116 and transcribed into fluorescent cRNA utilizing a random priming method (Arraystar Super RNA

117 Labeling Kit; Arraystar). The labeled cRNAs were hybridized onto the Arraystar Human

118 circRNA Array V2 $(8 \times 15 \mathrm{~K}$; Arraystar $)$. After washing the slides, the arrays were scanned using

119 the Agilent Scanner G2505C system (Agilent, Santa Clara, CA).

120

121

122

123

124

\section{Microarray data analysis}

Agilent Feature Extraction software (version 11.0.1.1) was used to analyze acquired array images. Quantile normalization and subsequent data processing were performed using the $\mathrm{R}$ software limma package. Differentially expressed circRNAs with statistical significance between two groups were identified through volcano plot filtering or fold change filtering. Hierarchical clustering was performed to show the distinguishable circRNA expression patterns among the samples. The interaction of circRNAs and miRNAs was predicted using Arraystar's homemade miRNA target prediction software based on TargetScan and miRanda. All the differentially expressed circRNAs were annotated in detail using the circRNA/miRNA interaction information.

\section{Functional analysis}


130 To further estimate the biological function of these candidate circRNAs, the targeted genes of

131 miRNAs that were predicted to interact with circRNAs were determined using TargetScan

132 (Enright et al. 2003) and miRanda (Pasquinelli et al. 2012). All miRNA gene targets were

133 identified by a p-value cutoff at 0.05 . These putative target genes were then checked for

134 functional enrichment using DAVID (Database for Annotation, Visualization and Integrated

135 Discovery) (Huang da et al. 2009), and KEGG (Kyoto Encyclopedia of Genes and Genomes)

136 analysis was used to identify the significant pathways involved. The circRNA-miRNA-mRNA

137 interaction network was constructed by Cytoscape (http://www.cytoscape.org/) (Shang et al.

138 2016).

\section{Reverse transcription and $\mathrm{QPCR}$ validation}

For circRNA analysis, total RNA was reverse transcribed using the FastQuant RT kit (Tangent Biotech, Beijing, China, KR106) and random primers. qRT-PCR was achieved using the Fast SYBR ${ }^{\circledR}$ Green Master Mix (Thermo Fisher Scientific (Waltham, MA, USA) in a StepOne Plus $^{\mathrm{TM}}$ Real-Time PCR System (Applied Biosystems) with a 20- $\mu \mathrm{L}$ PCR reaction mixture that included $2 \mu \mathrm{L}$ of cDNA, $10 \mu \mathrm{L}$ of Fast SYBRGreen Master Mix $(2 \times), 0.2 \mu \mathrm{L}$ of forward primer $(10 \mu \mathrm{M}), 0.2 \mu \mathrm{L}$ of reverse primer $(10 \mu \mathrm{M})$, and $7.6 \mu \mathrm{L}$ of nucleus-free water. The reactions were incubated in a MicroAmp ${ }^{\mathrm{TM}}$ Fast Optical 96 -well reaction plate at $95^{\circ} \mathrm{C}$ for $20 \mathrm{sec}$, followed by 40 cycles at $95^{\circ} \mathrm{C}$ for $5 \mathrm{sec}$ and $60^{\circ} \mathrm{C}$ for $34 \mathrm{sec}$. The melting curve with a single peak indicated the specific amplification of the expected fragments. The relative RNA expression level was calculated using the $\Delta \mathrm{Ct}$ method. Divergent primers rather than the 
150

151

152

commonly used convergent primers were designed for circular RNA amplification. The sequences of GAPDH and circRNAs primers are listed in Supplementary Table S1. All experiments were repeated three times.

\section{BRAFV600E mutation}

The BRAFV600E mutation was detected in the tumor tissues of these 40 PTC patients using allelic-specific primer PCR (ASP-PCR). A 201-bp fragment was amplified using a primer sequence designed for BRAF (Supplementary Table S1). The expected PCR products were separated on agarose gels and visualized by golden view staining, followed by Sanger sequencing validation.

\section{Statistical analysis}

All statistical data were analyzed using Statistical Product and Service Solutions SPSS software 19.0 (SPSS, Chicago, IL) and GraphPad Prism 5.0 (GraphPad Software, La Jolla, CA). The differences in expression of circRNAs between PTC and paired adjacent normal tissues were evaluated using Student's t test. The mean circRNA expression level was used as the cutoff value to define the high and low expression of circRNAs. The correlations between the circRNA levels and clinicopathological factors were analyzed by Student's t-test, Fisher's exact test, or nonparametric test. A receiver operating curve (ROC) was established to assess the diagnostic performance of hsa_circRNA_047771 and hsa_circRNA_007148. A P value $<0.05$ was considered significant. 


\section{Results}

\section{circRNA profiles in normal and tumor tissues}

To profile circRNA expression in PTC, we performed circRNA microarray analysis in PTC and matched noncancerous thyroid tissues. Hierarchical clustering analysis indicated that the expression patterns of circRNAs in PTC tissues were significantly different from those in normal tissues (Fig. 1A, fold change $>1.5, \mathrm{p}<0.05$ ). Red and green colors indicate the high and low expression levels of circRNAs, respectively. The red point of the volcano plot determined the significantly differentially expressed circRNAs between the normal and PTC samples with a fold change $>1.5$ (Fig. 1B). The scatter plot revealed the difference in circRNA expression in PTC tumor tissues and paired normal tissues (Fig. 1C). The points above and below the green lines represent 1.5-fold up and down of circRNAs between the normal and PTC samples, respectively. We found that 383 circRNAs were differentially expressed in PTC tissues compared with normal tissues. Among them, 206 circRNAs were up regulated and 177 circRNAs were downregulated (fold change $\geq 1.5, \mathrm{P}<0.05$ and FDR $<0.05$ ) in three paired carcinoma and normal tissues. The differently deregulated circRNAs were mostly located in exons (Fig. 1D). The top 10 differentially expressed circRNAs are listed in Table 2.

\section{Validation of dysregulated circRNAs}

According to the fold change tested in the microarray analysis, eight circRNAs (four upregulated and four downregulated) were selected for validation of the microarray results in 40 paired nontumorous and tumor samples by qRT-PCR. All four upregulated and four downregulated 
189

190

191

circRNAs were differentially expressed in PTC compared with those in normal tissues (Fig. 2A$\mathrm{H})$. These results were consistent with the microarray data, indicating the validation of these results.

\section{Functional analysis of differentially expressed circRNAs}

It is widely accepted that circRNAs are important transcriptional regulators of gene expression involved in the pathogenesis of various cancers. To explore the underlying mechanisms of dysregulated circRNAs involved in the tumorigenesis or development of PTC, Gene Ontology (GO) enrichment analysis of differentially expressed target genes was performed to evaluate the functional significance of these candidate circRNAs. The predominantly enriched GO items of upregulated circRNAs in PTC were correlated with cell communication-related genes (Fig. 3A), while the GO terms of downregulated circRNAs were mainly involved in the plasma membrane (Fig. 3B). These enriched terms, involving cell communication, signal transducer activity, RNA polymerase II transcription factor, and transcriptional activator activity, suggest that these circRNAs play important roles in cell-to-cell signaling and interaction, cellular proliferation, cellular function and maintenance.

KEGG analysis revealed that 50 pathways were significantly enriched among the target genes of upregulated circRNAs (Bonferroni $<0.05$ ), whereas 50 pathways were associated with downregulated circRNAs (Bonferroni $<0.05$ ). Notably, several important pathways were enriched, such as the VEGF signaling pathway, ras signaling pathway, and Notch signaling pathway (Fig. 4A, 4B). These findings suggest that circRNAs might play a significant role in 
PTC carcinogenesis and metastasis.

\section{Clinical implications of novel circRNAs in PTC}

211 Based on the validation data, hsa_circRNA_007148 and hsa_circRNA_047771 were the most

212 significantly elevated and decreased in PTC samples compared with those in normal samples.

213 Therefore, we evaluated their potential clinical value by analyzing the correlation of these two

214 candidate circRNAs with several clinicopathological parameters of PTC patients. Table 3 shows

215 that lower hsa_circRNA_047771 expression levels were associated with BRAFV600 mutation,

216 lymph node metastasis (LNM), and TNM stage (all $P<0.05)$. However, we observed no

217 association between the hsa_circRNA_047771 expression levels and other clinicopathological

218 factors, including sex, age, a history of Hashimoto's thyroiditis, thyroid imaging reporting and

219 data system (TIRAIDS), tumor size, and multifocality. We further found that a higher expression

220 level of hsa_circRNA_007148 was significantly correlated with LNM $(\mathrm{P}<0.05)$, while no other

221 clinicopathological factor was found to be significantly associated with the

222 hsa_circRNA_007148 expression level $(P>0.05)$. Additionally, similar associations were found

223 with the other six deregulated circRNAs (Supplementary Table S2). Next, we performed ROC

224 curve analysis to assess the diagnostic value of hsa_circRNA_047771 and hsa_circRNA_007148

225 in distinguishing PTC tissues from normal tissues. The areas under ROC curve were $0.876(95 \%$

226 CI, 0.78-0.94) for hsa_circRNA_047771 and 0.846 (95\% CI, 0.75-0.96) for

227 hsa_circRNA_007148 (Fig. 5A, 5B; Table 4). 
229 We next used Arraystar's circRNA target prediction software to identify potential miRNAs that 230 could bind to hsa_circRNA_047771 and hsa_circRNA_007148 via miRNA response elements 231 (MREs). The top 5 ranking MRE targets for the differentially expressed circRNAs are shown in 232 supplementary Figs. S1, 2. Notably, among the list of miRNAs targeted by 233 hsa_circRNA_047771, miR-522-3p/miR-153-5p were implicated in PTC in previous reports. We 234 further detected the expression levels of miR-522-3p/miR-153-5p in the 40 paired PTC and 235 normal samples. miR-522-3p/miR-153-5p were upregulated in PTC tissues compared with those in adjacent normal tissues (supplementary Fig. S3). These results provide powerful evidence that 237 hsa_circRNA_047771/ miR-522-3p/miR-153-5p could participate in the pathogenesis of PTC.

To fully understand the underlying mechanism between deregulated circRNAs and the biological progress of PTC, the target prediction program was performed to predict the potential target genes in the database. Based on the data of the deregulated circRNAs, their predicted MREs and targeted genes, we constructed a network map of cancer related circRNA-miRNA-mRNA interactions using Cytoscape that included 36 mRNAs and 34 miRNAs for

hsa_circRNA_047771 and 41 mRNAs and 51 miRNAs for hsa_circRNA_007148 (Fig. 6A, 6B). 
244

245

246

247

248

249

250

251

252

253

254

255

256

\section{Discussion}

Recently, the crucial role of epigenetic regulation in the development and pathogenesis of cancers has been widely recognized. There is abundant evidence suggesting that ncRNAs, including miRNAs, lncRNAs and circular RNAs, are strongly associated with the biological behavior of tumors. However, the role of circRNAs in PTC remains largely unknown. In the present study, we characterized the expression profile of circRNAs in PTC tissues and identified many aberrantly expressed circRNAs in PTC tissues, followed by validation of eight significantly different expressed circRNAs. Bioinformatics analysis indicated that deregulated circRNAs might be implicated in the biological process of PTC by regulating tumor-related pathways. The association between two novel circRNAs and aggressive clinicopathological characteristics provide evidence that circRNAs may serve as prognostic predictors for PTC patients. Additionally, the areas under ROC curve suggest that hsa_circRNA_047771 and hsa_circRNA_007148 may be candidate diagnostic biomarkers for PTC.

PTC accounts for a large proportion of thyroid cancer and needs to be better characterized and understood. Although the functions of circRNAs in carcinogenesis and cancer development have drawn great attention, studies focused on the role of circRNAs in PTC remain scarce. To our knowledge, only one study has focused on the relationship between circRNAs and PTC(Peng et al. 2017). This study performed circRNA microarray on 6 paired PTC tumors and contralateral normal tissues, as well as six benign thyroid nodules, to investigate the profile of circRNA expression in PTC and identified one significantly downregulated circRNA 
264 (hsa_circRNA_100395) that was associated with two tumor-associated miRNA clusters (miR-

265 141-3p and miR-200a-3p), revealing a potential role of circRNAs in PTC pathogenesis.

266 However, to date, no study has investigated the clinical value of circRNAs in PTC patients. The

267 association between circRNA expression and prognosis factors of PTC, such as tumor size,

268

269

270

271

272

273

274

275

276

277

278

279

280

281

282

283

tumor stage, LNM, and BRAF mutation, remains unclear. It is crucial to understand the

underlying mechanism of circRNAs regarding tumor behavior at the molecular level with a

larger sample. It will be of great importance to identify novel diagnostic, prognostic, and

therapeutic targets. Thus, we performed circular RNA array to characterize the circRNA

expression profile to comprehensively understand its clinical implications in PTC. Furthermore, similar to Nianchun Peng et al.(Peng et al. 2017), our study identified deregulated circRNAs. In the present study, KEGG and GO enrichment analyses identified many significant terms involved in cell events and several important cancer-related signaling pathways, such as the VEGF signaling pathway, ras signaling pathway, and Notch signaling pathway. Particularly, the ras signaling pathway was implicated in the development of PTC. These findings suggest that circRNAs might play a significant role in PTC carcinogenesis and metastasis.

Our study identified two novel circRNAs that were significantly associated with aggressive prognostic features of PTC, such as LNM and advanced TNM stage. These data indicated that deregulated circRNAs could predict the prognosis of patients with PTC. Interestingly, we found that a lower expression of hsa_circRNA_047771 is more likely to manifest with BRAFV600E mutation. BRAFV600E mutation has emerged as a poor prognostic marker for PTC patients. The 
284 clinical usefulness of BRAFV600E in the risk stratification, diagnosis and management of PTC

285 patients has been established(Elisei et al. 2008; Lupi et al. 2007). The predictive effect of

286 circRNAs in the prognosis of PTC patients and its association with BRAFV600E mutation in

287 PTC patients are considerably important and need further investigation.

The clinical application of circRNAs as potential molecular markers for the diagnosis and treatment of disease are promising(Meng et al. 2017), as confirmed by our results. circRNAs are highly stable and conservative in the cytoplasm and other body fluids, such as saliva and exosomes(Bahn et al. 2015; Dou et al. 2016). The observed high stability of circRNAs suggests that circRNAs will likely be a reliable biomarker for the preoperative diagnosis of PTC. Given the diagnostic limitations of FNAB in indeterminate cytology and insufficient sensitivity of molecular testing(Haugen et al. 2016; Li et al. 2015), the detection of circRNAs and clinical significance of circRNAs used for the preoperative diagnosis of thyroid cancers are attractive and critical issues related to thyroid cancer study that need to be well defined.

Circular RNAs are endogenous ncRNA molecules that function as regulators involved in various biological courses of cancer, such as development and proliferation(He et al. 2017; Xin et al. 2017). The mechanisms underlying circRNAs and tumorigenesis may be primarily associated with altered gene expression mediated by acting as an miRNA sponge(Chen et al. 2015). Recent studies have revealed that aberrant circRNA expression was closely correlated with various 
304

305

306

307

308

309

310

311

312

313

314

315

316

317

et al. 2017) found that circRNA_100290 could regulate oral squamous cell carcinomas through miR-29b-mediated inhibition of cell proliferation and the reduction of CDK6 expression. Another circRNA identified in a recent study, termed circHIPK3, was observed to be differentially expressed in several normal and tumor tissues and functions as a gene regulator by regulating miRNA activity(Zheng et al. 2016). These findings were confirmed in our study. We observed that each circRNA could bind to multiple miRNAs, some of which have been reported to be involved in PTC biology. For example, we found that miR-522-3p/miR-153-5p were targets of one novel down-expressed circRNA (hsa_circRNA_047771). These deregulated miRNAs have been reported to significantly correlate with thyroid cancer differentiation and progression(Boufraqech et al. 2016; Fuziwara \& Kimura 2016). Thus, further investigations are necessary to clarify the specific mechanism between deregulated circRNAs and PTC pathology.

Several limitations of this study must be addressed. First, the sample size was limited, and other thyroid carcinomas were not included in this study other than PTC. Second, we did not clarify the mechanism of circRNA/miRNA in the PTC pathological process. The molecular mechanism should be experimentally determined and elaborated in the future. Third, given the clinical applications of circRNAs, further studies will be required to evaluate the diagnostic value of circRNA levels in serum or FNAB samples.

\section{Conclusions}

The study demonstrated that several circRNAs are expressed differently in PTC and participate in tumor pathogenesis. Two PTC-associated circRNAs could serve as potential diagnostic 
324 markers and predict the prognosis of PTC patients. Furthermore, the study revealed that

325 circRNAs may have great potential as prognosis predictors and therapeutic targets for PTC

326 patients in the near future. 
327

328

329

330

331

332

333

334

335

336

337

338

339

340

341

342

343

344

\section{References}

Bahn JH, Zhang Q, Li F, Chan TM, Lin X, Kim Y, Wong DT, and Xiao X. 2015. The landscape of microRNA, Piwi-interacting RNA, and circular RNA in human saliva. Clin Chem 61:221-230. 10.1373/clinchem.2014.230433

Boufraqech M, Klubo-Gwiezdzinska J, and Kebebew E. 2016. MicroRNAs in the thyroid. Best Pract Res Clin Endocrinol Metab 30:603-619. 10.1016/j.beem.2016.10.001

Chen I, Chen CY, and Chuang TJ. 2015. Biogenesis, identification, and function of exonic circular RNAs. Wiley Interdiscip Rev RNA 6:563-579. 10.1002/wrna.1294

Chen L, Zhang S, Wu J, Cui J, Zhong L, Zeng L, and Ge S. 2017. circRNA_100290 plays a role in oral cancer by functioning as a sponge of the miR-29 family. Oncogene. 10.1038/onc.2017.89

Chen W, and Schuman E. 2016. Circular RNAs in Brain and Other Tissues: A Functional Enigma. Trends Neurosci 39:597-604. 10.1016/j.tins.2016.06.006

Dou Y, Cha DJ, Franklin JL, Higginbotham JN, Jeppesen DK, Weaver AM, Prasad N, Levy S, Coffey RJ, Patton JG, and Zhang B. 2016. Circular RNAs are down-regulated in KRAS mutant colon cancer cells and can be transferred to exosomes. Sci Rep 6:37982. $10.1038 /$ srep 37982

Elisei R, Ugolini C, Viola D, Lupi C, Biagini A, Giannini R, Romei C, Miccoli P, Pinchera A, and Basolo F. 2008. BRAF(V600E) mutation and outcome of patients with papillary thyroid carcinoma: a 15-year median follow-up study. J Clin Endocrinol Metab 93:3943- 
348

349

350

351

352

353

354

355

356

357

358

359

360

361

362

363

364

365

366

367

Enright AJ, John B, Gaul U, Tuschl T, Sander C, Marks DS: MicroRNA targets in Drosophila. Genome biology 2003; 5(1):R1.

Fuziwara CS, and Kimura ET. 2016. MicroRNAs in thyroid development, function and tumorigenesis. Mol Cell Endocrinol. 10.1016/j.mce.2016.12.017

Han D, Li J, Wang H, Su X, Hou J, Gu Y, Qian C, Lin Y, Liu X, Huang M, Li N, Zhou W, Yu Y, and Cao X. 2017. Circular RNA MTO1 acts as the sponge of miR-9 to suppress hepatocellular carcinoma progression. Hepatology. 10.1002/hep.29270

Haugen BR. 2017. 2015 American Thyroid Association Management Guidelines for Adult Patients with Thyroid Nodules and Differentiated Thyroid Cancer: What is new and what has changed? Cancer 123:372-381. 10.1002/cncr.30360

Haugen BR, Alexander EK, Bible KC, Doherty GM, Mandel SJ, Nikiforov YE, Pacini F, Randolph GW, Sawka AM, Schlumberger M, Schuff KG, Sherman SI, Sosa JA, Steward DL, Tuttle RM, and Wartofsky L. 2016. 2015 American Thyroid Association Management Guidelines for Adult Patients with Thyroid Nodules and Differentiated Thyroid Cancer: The American Thyroid Association Guidelines Task Force on Thyroid Nodules and Differentiated Thyroid Cancer. Thyroid 26:1-133. 10.1089/thy.2015.0020

He J, Xie Q, Xu H, Li J, and Li Y. 2017. Circular RNAs and cancer. Cancer Lett 396:138-144. 10.1016/j.canlet.2017.03.027

Hsiao KY, Lin YC, Gupta SK, Chang N, Yen L, Sun HS, and Tsai SJ. 2017. Noncoding Effects of Circular RNA CCDC66 Promote Colon Cancer Growth and Metastasis. Cancer Res 
369

370

371

372

373

374

375

376

377

378

379

380

381

382

383

384

385

386

387

388

Huang da W, Sherman BT, Lempicki RA: Systematic and integrative analysis of large gene lists using DAVID bioinformatics resources. Nature protocols 2009; 4(1):44-57.

Jeck WR, and Sharpless NE. 2014. Detecting and characterizing circular RNAs. Nat Biotechnol 32:453-461. 10.1038/nbt.2890

Kwak JY, Kim EK, Chung WY, Moon HJ, Kim MJ, and Choi JR. 2009. Association of BRAFV600E mutation with poor clinical prognostic factors and US features in Korean patients with papillary thyroid microcarcinoma. Radiology 253:854-860. 10.1148/radiol.2533090471

La Vecchia C, Malvezzi M, Bosetti C, Garavello W, Bertuccio P, Levi F, and Negri E. 2015. Thyroid cancer mortality and incidence: a global overview. Int J Cancer 136:2187-2195. $10.1002 /$ ijc. 29251

Li F, Chen G, Sheng C, Gusdon AM, Huang Y, Lv Z, Xu H, Xing M, and Qu S. 2015. BRAFV600E mutation in papillary thyroid microcarcinoma: a meta-analysis. Endocr Relat Cancer 22:159-168. 10.1530/erc-14-0531

Liyanarachchi S, Li W, Yan P, Bundschuh R, Brock P, Senter L, Ringel MD, de la Chapelle A, and He H. 2016. Genome-Wide Expression Screening Discloses Long Noncoding RNAs Involved in Thyroid Carcinogenesis. J Clin Endocrinol Metab 101:4005-4013. 10.1210/jc.2016-1991

Lu L, Sun J, Shi P, Kong W, Xu K, He B, Zhang S, and Wang J. 2017. Identification of circular RNAs as a promising new class of diagnostic biomarkers for human breast cancer. 
Lukiw WJ. 2013. Circular RNA (circRNA) in Alzheimer's disease (AD). Front Genet 4:307.

Lupi C, Giannini R, Ugolini C, Proietti A, Berti P, Minuto M, Materazzi G, Elisei R, Santoro M, Miccoli P, and Basolo F. 2007. Association of BRAF V600E mutation with poor

Pasquinelli AE: MicroRNAs and their targets: recognition, regulation and an emerging reciprocal relationship. Nature reviews Genetics 2012; 13(4):271-282.

Peng N, Shi L, Zhang Q, Hu Y, Wang N, and Ye H. 2017. Microarray profiling of circular RNAs in human papillary thyroid carcinoma. PLoS One 12:e0170287. 10.1371/journal.pone.0170287

Rybak-Wolf A, Stottmeister C, Glazar P, Jens M, Pino N, Giusti S, Hanan M, Behm M, Bartok O, Ashwal-Fluss R, Herzog M, Schreyer L, Papavasileiou P, Ivanov A, Ohman M, Refojo D, Kadener S, and Rajewsky N. 2015. Circular RNAs in the Mammalian Brain Are Highly Abundant, Conserved, and Dynamically Expressed. Mol Cell 58:870-885. 10.1016/j.molcel.2015.03.027

Shang X, Li G, Liu H, Li T, Liu J, Zhao Q, Wang C: Comprehensive Circular RNA Profiling 
Reveals That hsa_circ_0005075, a New Circular RNA Biomarker, Is Involved in Hepatocellular Crcinoma Development. Medicine 2016; 95(22):e3811.

412 Siegel RL, Miller KD, and Jemal A. 2017. Cancer Statistics, 2017. CA Cancer J Clin 67:7-30.

414 Wu ZS, Wu Q, Wang CQ, Wang XN, Wang Y, Zhao JJ, Mao SS, Zhang GH, Zhang N, and Xu XC. 2010. MiR-339-5p inhibits breast cancer cell migration and invasion in vitro and may be a potential biomarker for breast cancer prognosis. BMC Cancer 10:542. 10.1186/1471-2407-10-542

Xing M. 2007. BRAF mutation in papillary thyroid cancer: pathogenic role, molecular bases, and

Xin Z, Ma Q, Ren S, Wang G, and Li F. 2017. The understanding of circular RNAs as special triggers in carcinogenesis. Brief Funct Genomics 16:80-86. 10.1093/bfgp/elw001

Zhao ZJ, and Shen J. 2015. Circular RNA participates in the carcinogenesis and the malignant behavior of cancer. RNA Biol:1-8. 10.1080/15476286.2015.1122162

Zheng Q, Bao C, Guo W, Li S, Chen J, Chen B, Luo Y, Lyu D, Li Y, Shi G, Liang L, Gu J, He X, and Huang S. 2016. Circular RNA profiling reveals an abundant circHIPK3 that regulates cell growth by sponging multiple miRNAs. Nat Commun 7:11215. $10.1038 /$ ncomms 11215 
428

429

430

431

432

433

434

435

436

437

438

439

440

441

442

443

444

445

446

447

\section{Figure legends}

\section{Fig. 1. circRNA expression profile in papillary thyroid carcinoma and paired normal}

tissues. (A) Heat map from the microarray analysis of circRNA expression in normal and papillary thyroid carcinoma (PTC) tissues (fold change $>1.5, \mathrm{p}<0.05$ ). Red and green colors indicate high and low expression levels, respectively. (B) Volcano plot constructed to visualize the differentially expressed circRNAs between PTC and adjacent normal tissues. The vertical lines indicate 1.5-fold upregulation and downregulation. The horizontal line represents a P-value of 0.05 . The red points in the plot represent the statistically deregulated circRNAs. (C) Scatter plots represent the differential expression of circRNAs in PTC tumors (group-C) versus normal thyroid tissue (group-N). The values of the $\mathrm{x}$ - and y-axes in the scatter plot were the normalized signal intensities of the samples ( $\log 2$ scaled). The green lines represent the 1.5 -fold up and down of circRNAs between the normal and PTC samples. C: PTC tissue. N: adjacent normal tissue. (D) the proportion of differentially expressed circRNAs according to the different genomic locus (exonic, intronic, antisense, intragenic, and intergenic).

Fig. 2. Identification and $q R T-P C R$ validation of differentially expressed circRNAs. (A-D), (E-H) The relative expression of four upregulated and four downregulated circRNAs was validated in 40 human PTC tissues and adjacent non-tumorous tissues. ${ }^{* * *} P<0.001$. Normal: adjacent non-tumorous tissue; PTC: papillary thyroid tumor tissue. $\Delta \mathrm{Ct}$ values were used to assess the relative gene expression, which was normalized to the GAPDH expression level.

Fig. 3. Enrichment GO analysis of circRNA-target genes. Top 10 significant enriched GO 
448 terms of upregulated circRNAs(A) and downregulated circRNAs (B).

449 Fig. 4. Enrichment KEGG analysis of circRNA-target genes. Top 10 pathway terms in KEGG 450 pathway analysis of upregulated circRNAs (A) and downregulated circRNAs (B).

451 Fig. 5. Correlation of hsa_circRNA_047771 and hsa_circRNA_007148 with the diagnosis of 452 papillary thyroid carcinoma patients. Receiver operating characteristics (ROC) curve analysis 453 for hsa_circRNA_047771(A) and hsa_circRNA_007148) (B) according to their relative 454 expression level as measured by qPCR $(P<0.01)$.

455

456

Fig. 6. Construction of the circRNA-miRNA-mRNA network. (A) The network map contained the significantly upregulated hsa_circRNA_007148 (represented by brown nodes) along with their 51 downstream miRNAs (represented by red nodes) and 41 target mRNAs (represented by light-blue nodes). (B) The network map contained significantly downregulated hsa_circRNA_047771 (represented by brown nodes) along with their 34 downstream miRNAs (represented by red nodes) and target 36 mRNAs (represented by light-blue nodes). 


\title{
Figure 1
}

\section{circRNAs expression profile in papillary thyroid carcinoma and paired normal tissues.}

\begin{abstract}
(A) Heat map from the microarray analysis of circRNA expression in normal and $p$ apillary thyroid carcinoma (PTC) tissues (fold change $>1.5, p<0.05$ ). Red and green colors indicate high and low expression levels, respectively. (B) Scatter plots represent the differential expression of circRNAs in PTC tumors (group-C) versus normal thyroid tissue (group- $\mathrm{N}$ ). The values of the $\mathrm{x}$ - and $\mathrm{y}$-axes in the scatter plot were the normalized signal intensities of the samples (log2 scaled). The green lines represent the 1.5 -fold up and down of circRNAs between the normal and PTC samples. C: PTC tissue. N: adjacent normal tissue. (C) Volcano plot constructed to visualize the differentially expressed circRNAs between PTC and adjacent normal tissues. The vertical lines indicate 1.5 -fold upregulation and downregulation. The horizontal line represents a $P$-value of 0.05 . The red points in the plot represent the statistically deregulated circRNAs. (D) the proportion of differentially expressed circRNAs according to the different genomic locus (exonic, intronic, antisense, intragenic, and intergenic).
\end{abstract}


A
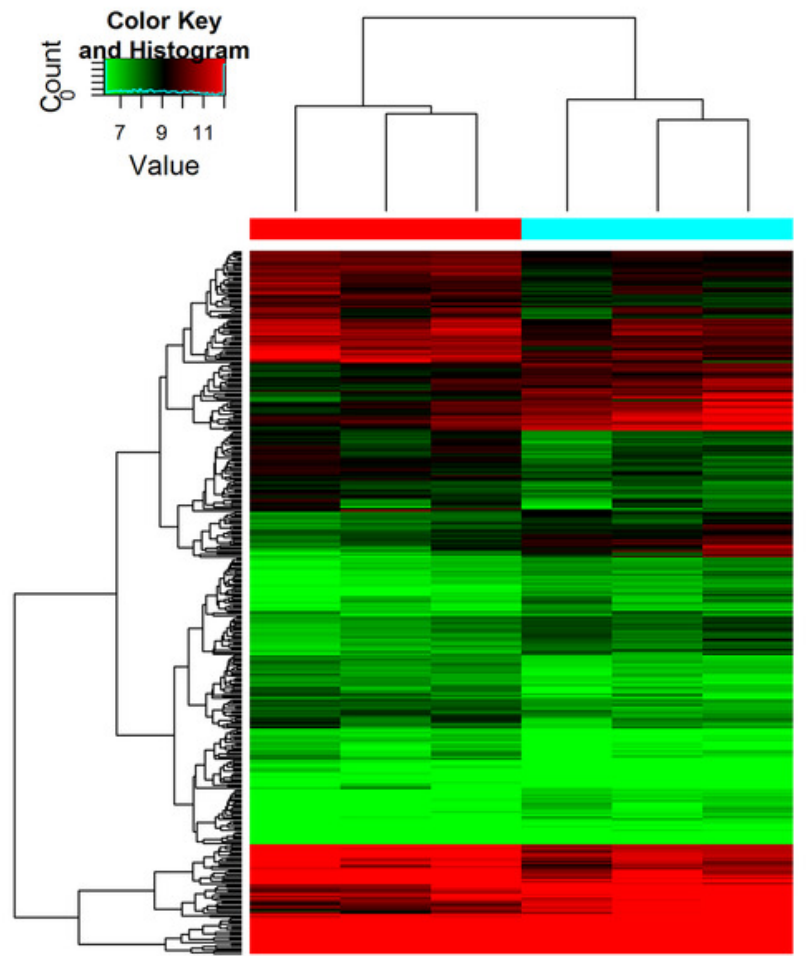

C

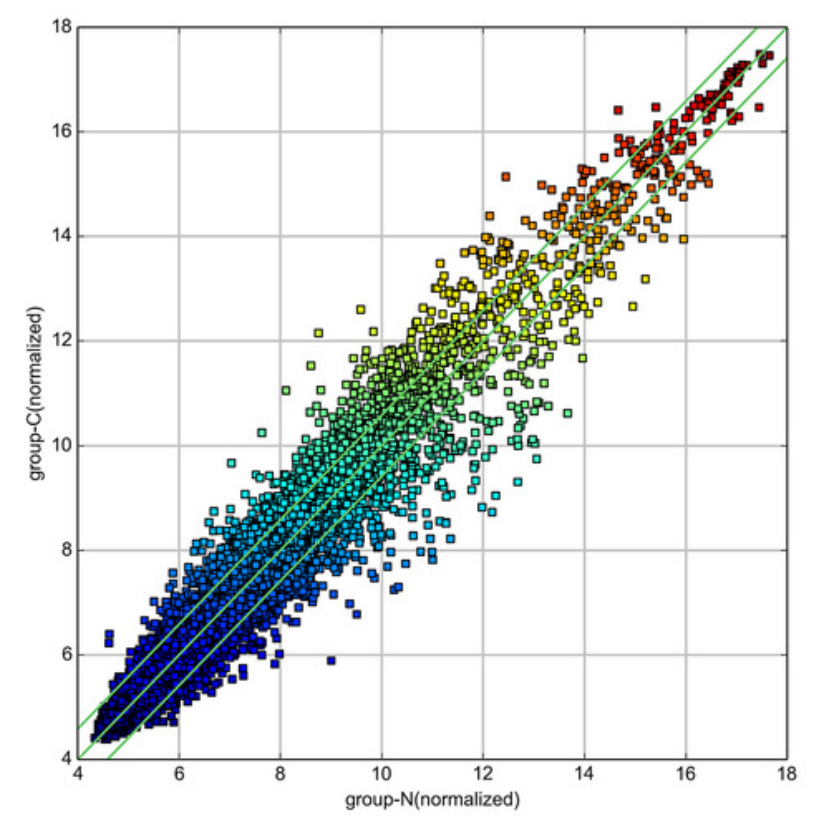

B

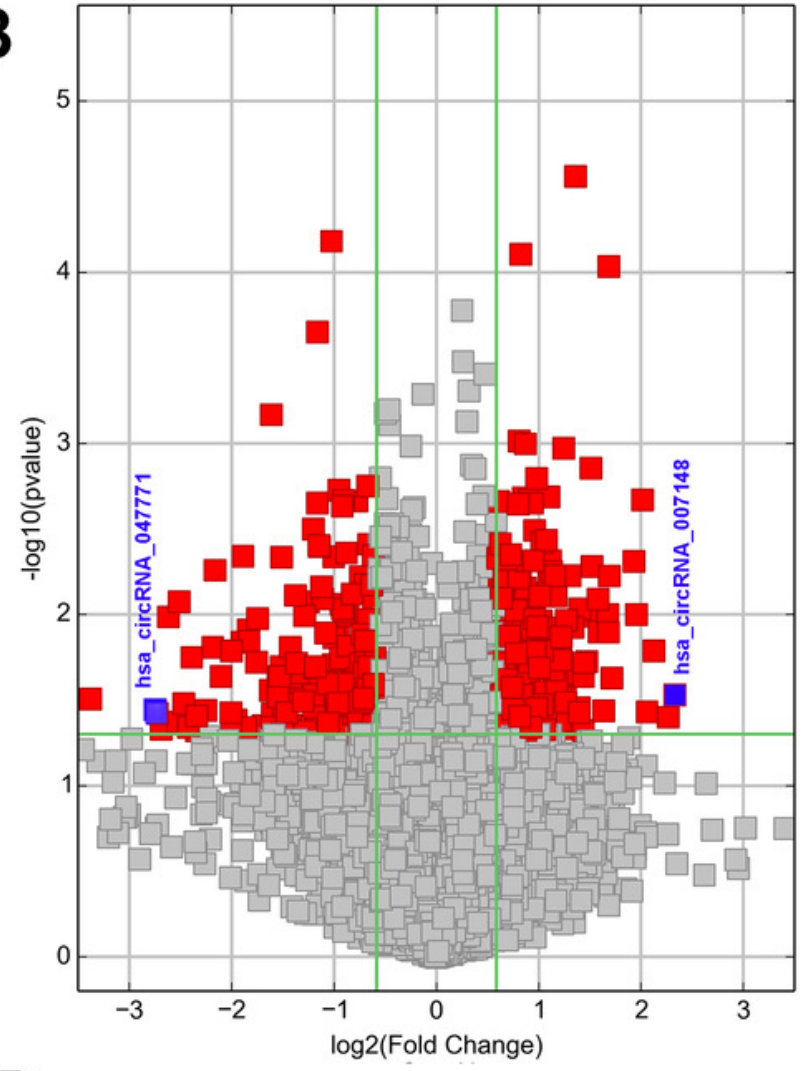

D

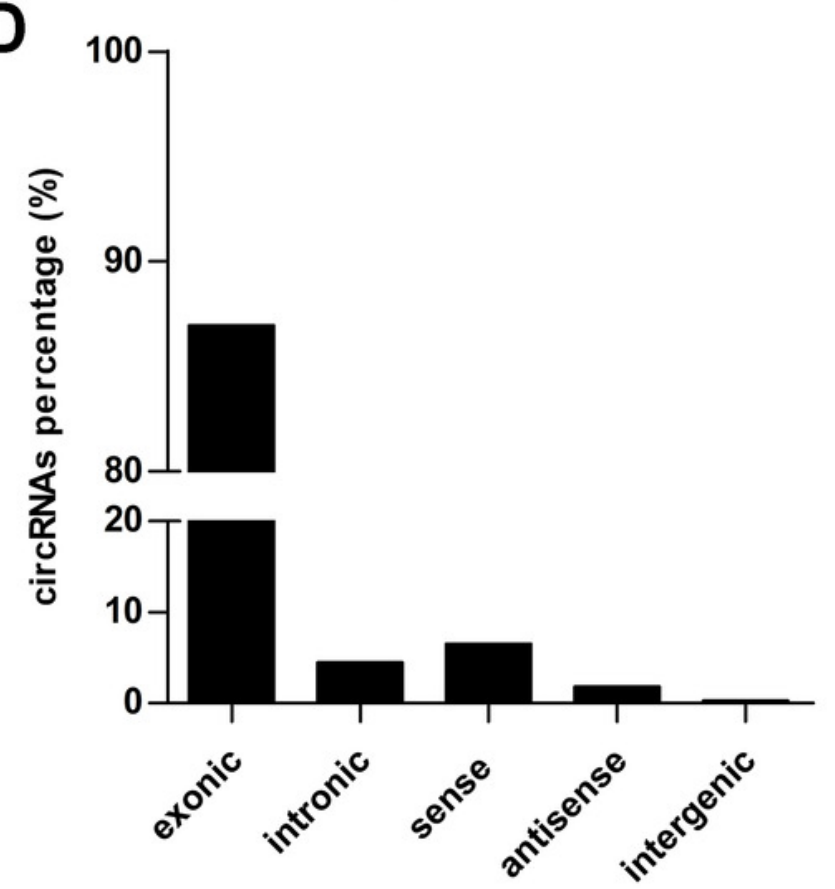


Figure 2

Identification and qRT-PCR validation of differentially expressed circRNAs.

(A-D), (E-H) The relative expression of four upregulated and four downregulated circRNAs was validated in 40 human PTC tissues and adjacent non-tumorous tissues. ${ }^{* * *} P<0.001$. Normal: adjacent non-tumorous tissue; PTC: papillary thyroid tumor tissue. $\Delta C$ Ct values were used to assess the relative gene expression, which was normalized to the GAPDH expression level.

A

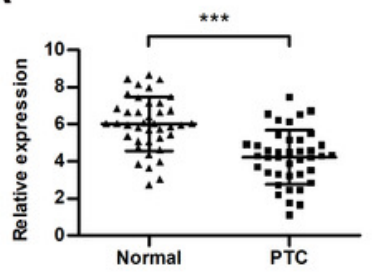

hsa_circRNA_007148

E

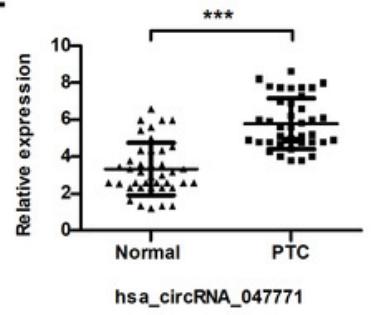

B

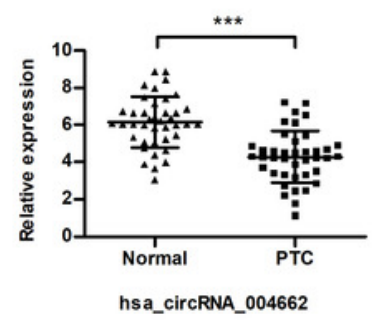

$\mathbf{F}$

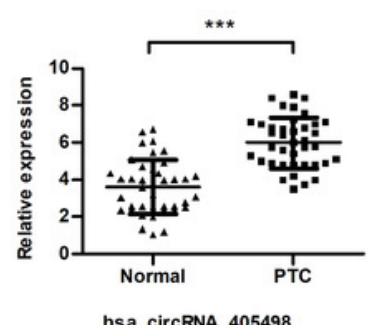

C

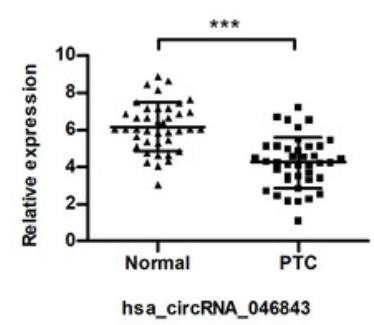

G

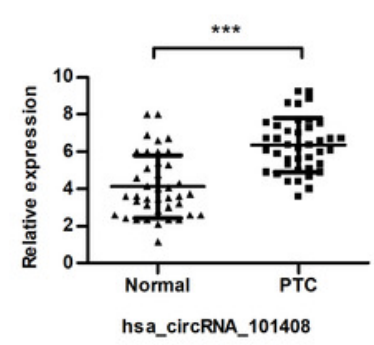

D

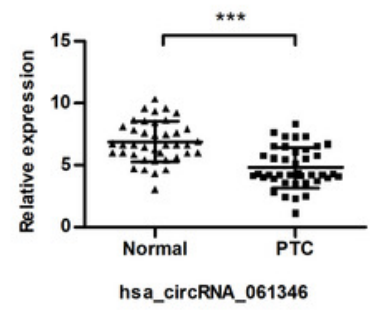

H

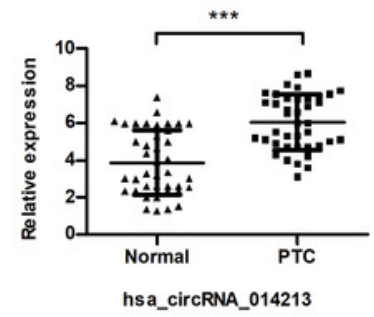


Figure 3

Enrichment analysis of circRNA-target genes.

Top 10 significant enriched $G 0$ terms of upregulated $\operatorname{circRNAs}(A)$ and downregulated circRNAs (B). 
A
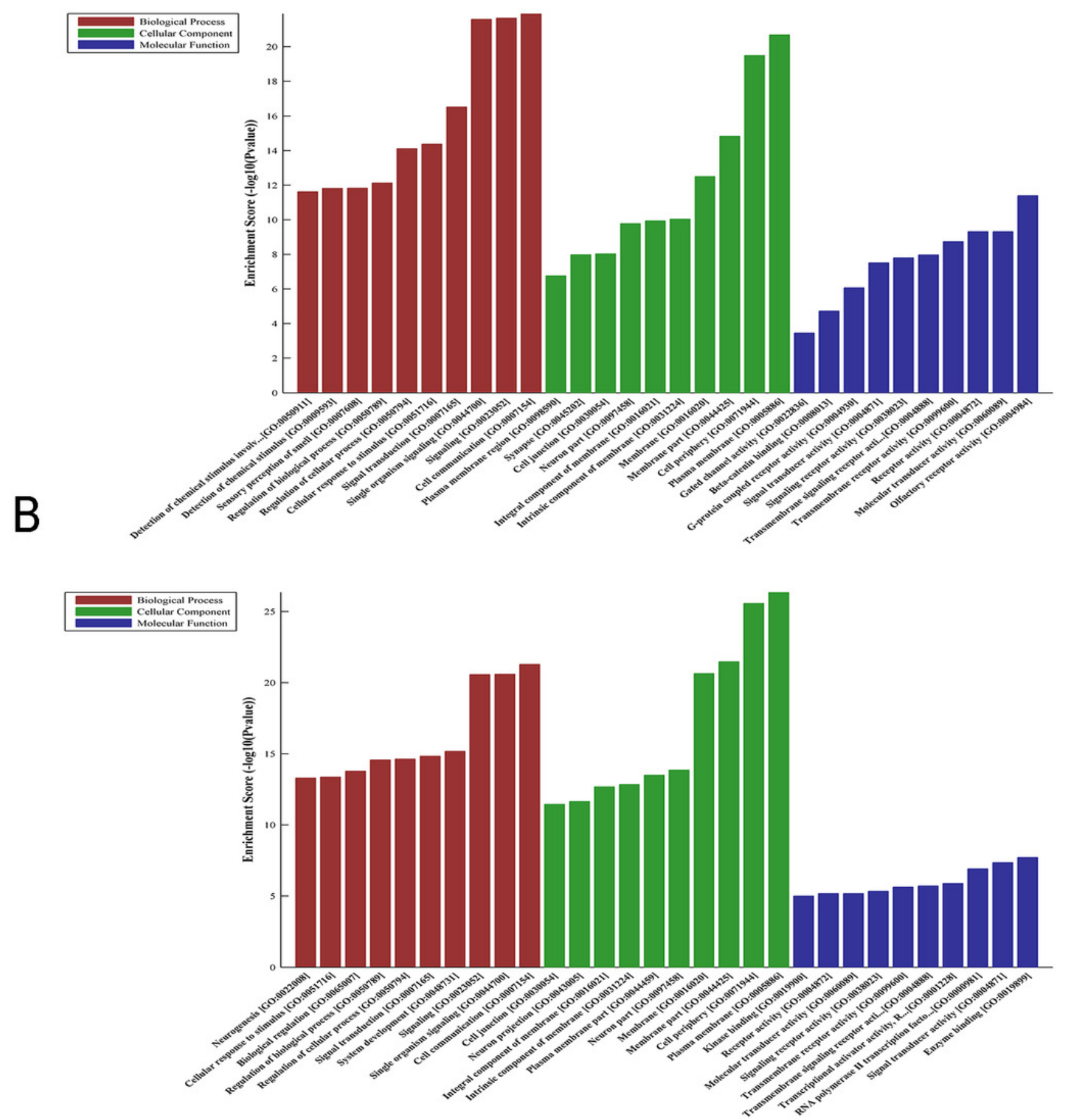


\section{Figure 4}

\section{Enrichment KEGG analysis of circRNA-target genes.}

Top 10 pathway terms in KEGG pathway analysis of upregulated circRNAs (A) and downregulated circRNAs (B).
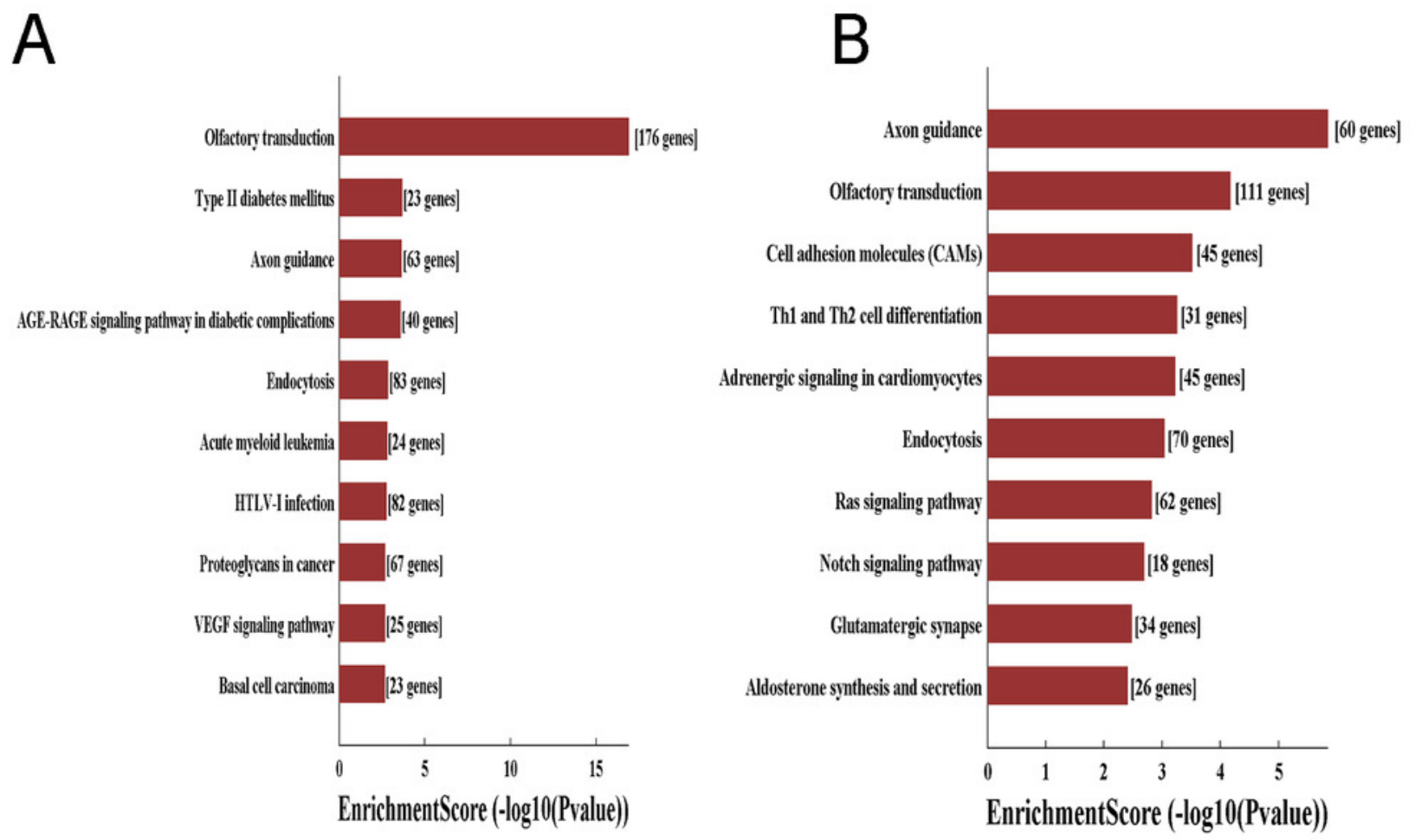
Figure 5

Correlation of hsa_circRNA_047771 and hsa_circRNA_007148 with the diagnosisof papillary thyroid carcinoma patients.

Receiver operating characteristics (ROC) curve analysis for hsa_circRNA_047771(A) and hsa_circRNA_007148) (B) according to their relative expression level as measured by qPCR $(P<0.01)$.
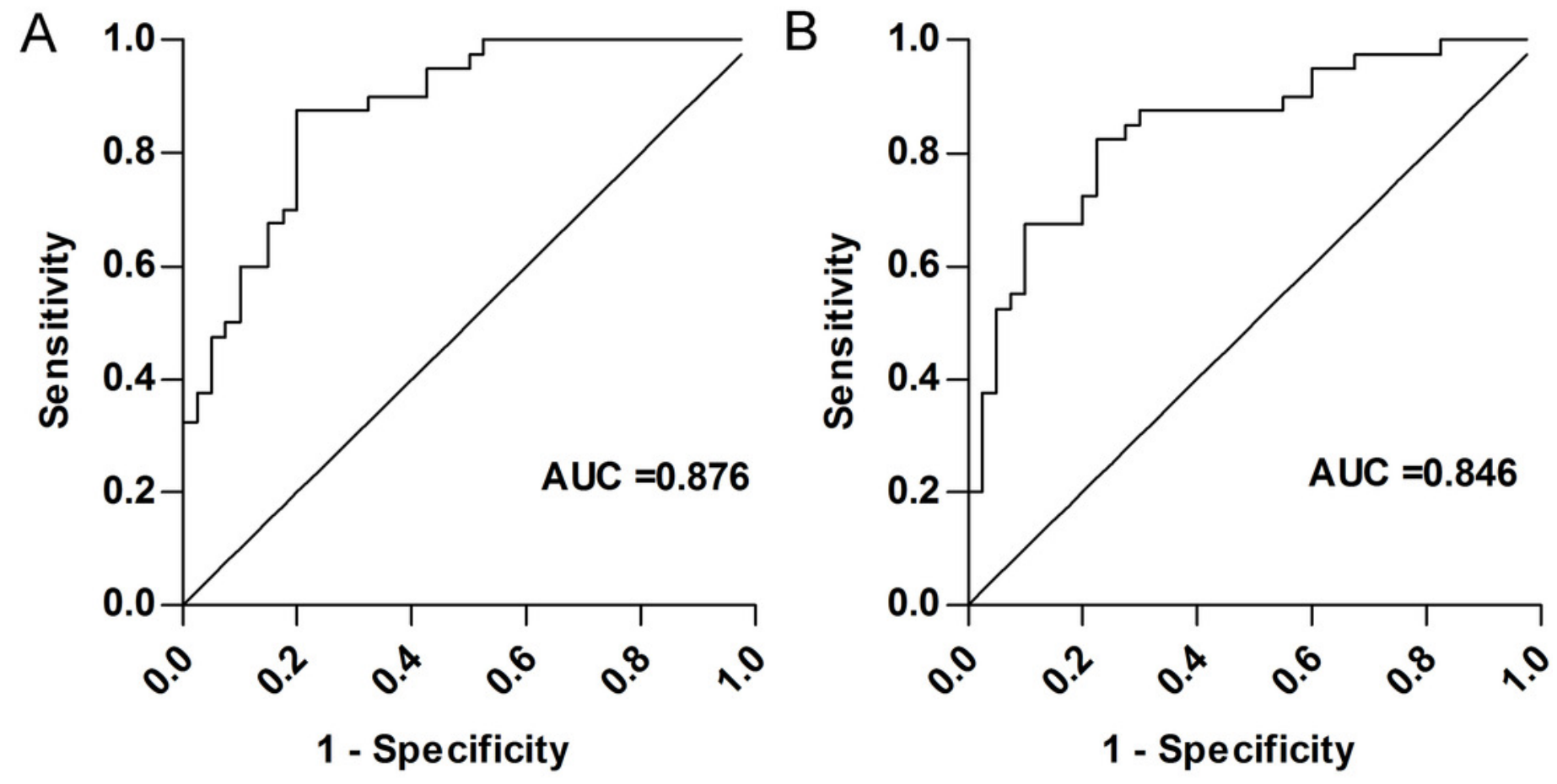

1 - Specificity 
Figure 6

\section{Construction of the circRNA-miRNA-mRNAnetwork .}

(A) The network map contained the significantly upregulated hsa_circRNA_007148 (represented by brown nodes) along with their 51 downstream miRNAs (represented by red nodes) and 41 target mRNAs (represented by light-blue nodes). (B) The network map contained significantly downregulated hsa_circRNA_ 047771 (represented by brown nodes) along with their 34 downstream miRNAs (represented by red nodes) and target 36 mRNAs (represented by light-blue nodes).

A

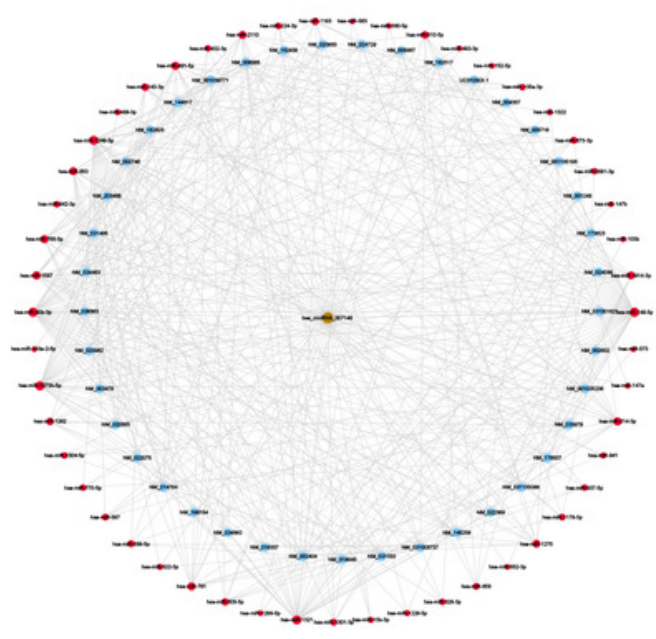

B

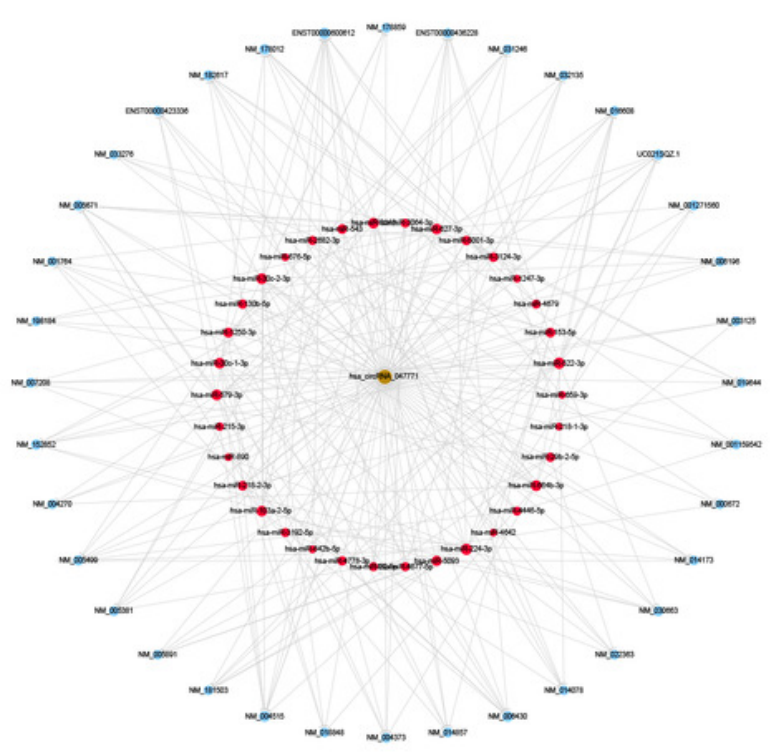




\section{Table $\mathbf{1}$ (on next page)}

Clinicopathological characteristics of participating patients.

Data are expressed as mean (sd) or $n$ (percent) or median (interquartile range); TIRAIDS, thyroid imaging reporting and data system; TNM, tumor-node-metastasis. LNM, lymph node metastasis. 
1 Table 1. Clinicopathological characteristics of participates patients.

\begin{tabular}{|c|c|}
\hline & Patients $(n=40)$ \\
\hline Age(years) & $43.5(13.1)$ \\
\hline$\geq 45$ & $21(52.5)$ \\
\hline$<45$ & $19(47.5)$ \\
\hline \multicolumn{2}{|l|}{ Sex } \\
\hline Female & $30(75)$ \\
\hline male & $10(25)$ \\
\hline \multicolumn{2}{|l|}{ Hashimoto } \\
\hline Yes & $6(15)$ \\
\hline No & $34(85)$ \\
\hline \multicolumn{2}{|l|}{ TIRAID } \\
\hline $4 a$ & $4(10)$ \\
\hline $4 b$ & $13(32.5)$ \\
\hline $4 c$ & $23(57.5)$ \\
\hline \multicolumn{2}{|l|}{ BRAF mutation } \\
\hline Yes & $16(40)$ \\
\hline No & $24(60)$ \\
\hline Tumor size (cm) & $1.2(0.8-2)$ \\
\hline \multicolumn{2}{|l|}{ TNM stage } \\
\hline I & $25(62.59)$ \\
\hline II & 0 \\
\hline III & $9(22.5)$ \\
\hline IV & $6(15)$ \\
\hline \multicolumn{2}{|l|}{ LNM } \\
\hline Yes & $18(45)$ \\
\hline No & $22(55)$ \\
\hline \multicolumn{2}{|l|}{ Focality } \\
\hline Unifocal & $13(32.5)$ \\
\hline Multifocal & $27(67.5)$ \\
\hline
\end{tabular}

2 Data are expressed as mean (sd) or $\mathrm{n}$ (percent) or median (interquartile

3 range); TIRAIDS, thyroid imaging reporting and data system; TNM,

4 tumor-node-metastasis. LNM, lymph node metastasis 


\section{Table 2 (on next page)}

Top 10 most upregulated and downregulated circRNAs in PTC tumors versus paired normal thyroid tissue. 
1 Table 2. Top 10 most upregulated and downregulated circRNAs in PTC tumors versus paired normal thyroid tissue.

\begin{tabular}{lllllll}
\hline circRNA & FC & P value & Type & chrom & GeneSymbol & miRNA binding sites \\
\hline $\begin{array}{l}\text { upregulated } \\
\text { hsa_circRNA_007148 }\end{array}$ & 5.014 & 0.02933 & exonic & chr3 & FNDC3B & hsa-miR-6785-5p,hsa-miR-1275,hsa-miR-490-3p,hsa-miR-1229-5p,hsa-miR-6088 \\
hsa_circRNA_004662 & 4.80 & 0.03966 & exonic & chr6 & SOD2 & hsa-miR-4520-2-3p,hsa-miR-208b-5p,hsa-miR-4713-5p,hsa-miR-4659b-3p \\
hsa_circRNA_046843 & 4.36 & 0.01622 & exonic & chr18 & ANKRD12 & hsa-miR-4524a-5p,hsa-miR-4753-3p,hsa-miR-3911,hsa-miR-876-5p,hsa-miR-942-5p \\
hsa_circRNA_103514 & 4.16 & 0.03694 & exonic & chr3 & FNDC3B & hsa-miR-147b,hsa-miR-510-5p,hsa-miR-15b-3p,hsa-miR-1323,hsa-miR-552-3p \\
hsa_circRNA_061346 & 4.03 & 0.00213 & exonic & chr21 & APP & hsa-miR-4778-3p,hsa-miR-5196-3p,hsa-miR-5193,hsa-miR-877-3p,hsa-miR-103a-2- \\
downregulated & & & & & & \\
hsa_circRNA_404959 & 10.40 & 0.03101 & exonic & chr12 & CLSTN3 & hsa-miR-6848-5p,hsa-miR-6081,hsa-miR-6720-5p,hsa-miR-5591-5p,hsa-miR-6165 \\
hsa_circRNA_047771 & 6.71 & 0.03596 & exonic & chr18 & NARS & hsa-miR-522-3p,hsa-miR-224-3p,hsa-miR-4677-5p,hsa-miR-140-5p,hsa-miR-153-5p \\
hsa_circRNA_405498 & 6.63 & 0.03760 & exonic & chr16 & GCSH & hsa-miR-6754-5p,hsa-miR-6812-5p,hsa-miR-5572,hsa-miR-6855-5p,hsa-miR-3612 \\
hsa_circRNA_101408 & 6.45 & 0.04666 & exonic & chr14 & TTLL5 & hsa-miR-136-5p,hsa-miR-500a-5p,hsa-miR-578,hsa-miR-34b-5p,hsa-miR-449c-5p \\
hsa_circRNA_014213 & 6.15 & 0.01028 & exonic & chr1 & SPRR1B & hsa-miR-6812-5p,hsa-miR-6819-5p,hsa-miR-608,hsa-miR-5572,hsa-miR-5088-5p \\
\hline
\end{tabular}

circRNA, circular RNA; PTC, papillary thyroid carcinoma; FC, fold change; Chrom: chromosome; miRNA: microRNA. 


\section{Table 3(on next page)}

Association between hsa_circRNA_047771 and has_circRNA_007148 expression levels and clinicopathological characteristics in40 PTC patients

\# The mean expression value was used as the cutoff scores for the high-expression and low-expression of circRNAs. Data are expressed as n (percent) or median (interquartile range); circRNA, circular RNA; PTC, papillary thyroid carcinoma; TIRAIDS, thyroid imaging reporting and data system; TNM, tumor-nodemetastasis; LNM, lymph node metastasis . ${ }^{*} P<0.05,{ }^{* *} P<0.01$. 
1 Table 3. Association between hsa_circRNA_047771 and hsa_circRNA_007148 expression levels and

2 clinicopathological characteristics in 40 PTC patients

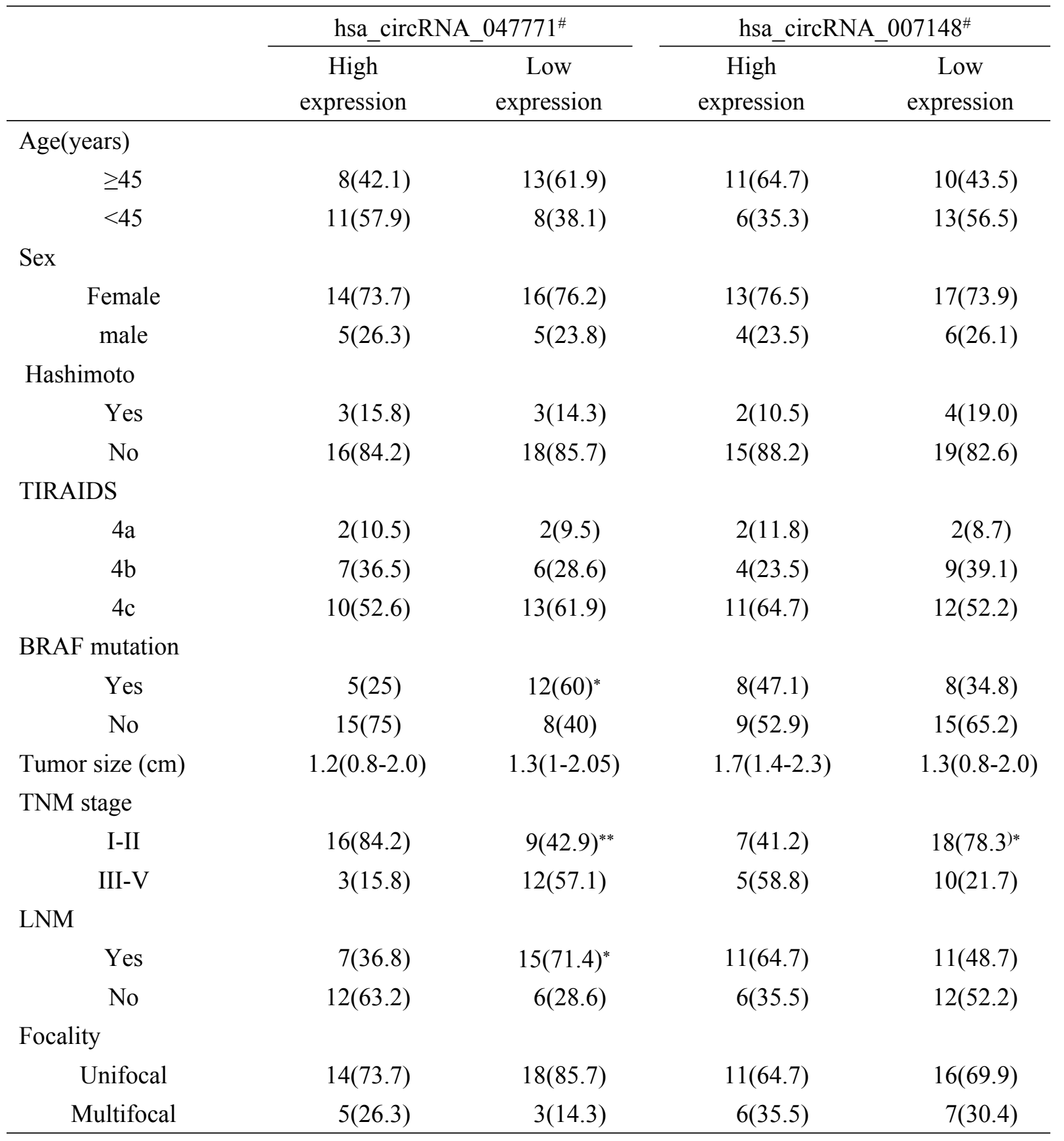

$3 \quad$ \#The mean expression value was used as the cutoff scores for the high-expression and low-expression of 4 circRNAs. Data are expressed as n (percent) or median (interquartile range); circRNA, circular RNA;

5 PTC, papillary thyroid carcinoma; TIRAIDS, thyroid imaging reporting and data system; TNM, tumor6 node-metastasis; LNM, lymph node metastasis. ${ }^{*} P<0.05,{ }^{* *} P<0.01$. 


\section{Table 4(on next page)}

Diagnostic value of circRNAs in papillary thyroid cancer.

The relative expression of each circRNA was calculated using the $\triangle \mathrm{Ct}$ method. PV, predictive value; AUC, area under the curve; $\mathrm{Cl}$, confidence interval. 
1 Table 4. Diagnostic value of circRNAs in papillary thyroid cancer.

\begin{tabular}{ccc}
\hline & hsa_circRNA_047771 & hsa_circRNA_007148 \\
\hline Sensitivity(95\% CI) & $87.5(0.73-0.96)$ & $82.5(0.63-0.97)$ \\
Specificity(95\% CI) & $80.0(0.64-0.91)$ & $77.5(0.62-0.89)$ \\
+PV (95\% CI) & $81.4(0.66-0.92)$ & $78.6(0.63-0.90)$ \\
-PV(95\% CI) & $86.5(0.71-0.96)$ & $81.6(0.66-0.92)$ \\
Youden index & 0.675 & 0.6 \\
AUC $(95 \% \mathrm{CI})$ & $0.876(0.78-0.94)$ & $0.846(0.75-0.96)$ \\
\hline
\end{tabular}

2 The relative expression of each circRNA was calculated using the $\Delta \mathrm{Ct}$ method. PV, predictive value;

3 AUC, area under the curve; CI, confidence interval. 